\title{
Frequency dependence of the radiative decay rate of excitons in self-assembled quantum dots: Experiment and theory
}

Stobbe, Søren; Johansen, Jeppe; Kristensen, Philip Trøst; Hvam, Jørn Märcher; Lodahl, Peter

Published in:

Physical Review B Condensed Matter

Link to article, DOI:

10.1103/PhysRevB.80.155307

Publication date:

2009

Document Version

Publisher's PDF, also known as Version of record

Link back to DTU Orbit

Citation (APA):

Stobbe, S., Johansen, J., Kristensen, P. T., Hvam, J. M., \& Lodahl, P. (2009). Frequency dependence of the radiative decay rate of excitons in self-assembled quantum dots: Experiment and theory. Physical Review $B$ Condensed Matter, 80(15), 155307. https://doi.org/10.1103/PhysRevB.80.155307

\section{General rights}

Copyright and moral rights for the publications made accessible in the public portal are retained by the authors and/or other copyright owners and it is a condition of accessing publications that users recognise and abide by the legal requirements associated with these rights.

- Users may download and print one copy of any publication from the public portal for the purpose of private study or research.

- You may not further distribute the material or use it for any profit-making activity or commercial gain

- You may freely distribute the URL identifying the publication in the public portal 


\title{
Frequency dependence of the radiative decay rate of excitons in self-assembled quantum dots: Experiment and theory
}

\author{
S. Stobbe, ${ }^{*}$ J. Johansen, P. T. Kristensen, J. M. Hvam, and P. Lodahl ${ }^{\dagger}$ \\ DTU Fotonik, Department of Photonics Engineering, Technical University of Denmark, Building 343, DK-2800 Kgs. Lyngby, Denmark
}

(Received 2 February 2009; revised manuscript received 4 September 2009; published 5 October 2009)

\begin{abstract}
We analyze time-resolved spontaneous emission from excitons confined in self-assembled InAs quantum dots placed at various distances to a semiconductor-air interface. The modification of the local density of optical states due to the proximity of the interface enables unambiguous determination of the radiative and nonradiative decay rates of the excitons. From measurements at various emission energies, we obtain the frequency dependence of the radiative decay rate, which is only revealed due to the separation of the radiative and nonradiative parts. It contains detailed information about the dependence of the exciton wave function on quantum dot size. We derive the quantum optics theory of a solid-state emitter in an inhomogeneous environment and compare this theory to our experimental results. Using this model, we extract the frequency dependence of the overlap between the electron and hole wave functions. We furthermore discuss three models of quantum dot strain and compare the measured wave-function overlap to these models. The observed frequency dependence of the wave-function overlap can be understood qualitatively in terms of the different compressibility of electrons and holes originating from their different effective masses and binding energies.
\end{abstract}

DOI: 10.1103/PhysRevB.80.155307

PACS number(s): 78.67.Hc, 42.50.Ct, 78.47.Cd

\section{INTRODUCTION}

Semiconductor quantum dots (QDs) are nanoscale solidstate structures that provide three-dimensional quantum confinement of otherwise mobile charge carriers. Self-assembled QDs of InAs embedded in GaAs provide confinement for both electrons and holes in a direct band-gap semiconductor. Hence, they are optically active with the benefits of a high quantum efficiency and compatibility with existing semiconductor technology. These properties make the QDs highly promising light sources for novel optical devices including optical quantum information devices. ${ }^{1}$ This has led to an increasing interest in the quantum-optical properties of QDs, and major achievements include the demonstration of the Purcell effect for QDs in solid-state cavities ${ }^{2}$ and strong coupling between a single QD and the optical mode of a cavity. ${ }^{3,4}$ Very recently also electrical tuning of such quantum photonics devices was demonstrated, ${ }^{5,6}$ which is a significant milestone toward practical all-solid-state cavity quantum electrodynamics devices.

Despite the recent progress, a thorough understanding of the dynamics of light-matter coupling for QDs in nanostructured photonic media is still lacking. Such an understanding is required for quantitative comparisons between experiment and theory. The problem is twofold, i.e., a detailed understanding of both the optical part and the electronic part is required. The optical part is described by the local density of optical states (LDOS) expressing the distribution of modes that the QD can radiate to, while the electronic part is determined by the exciton wave function for the QD. Here we will investigate an optical system, where the LDOS can be calculated exactly, and use that to extract detailed information about the QD. Our experimental results are compared to a theoretical QD model, and the effect of QD size, material composition, and strain is investigated. Such quantitative comparisons of experimental data to simple theoretical QD models are much needed in order to assess the full potential of QDs in nanostructured media for, e.g., single-photon sources, $, 7,8$ low-threshold lasers, ${ }^{9}$ or spontaneous emission control. ${ }^{10,11}$

When interpreting spontaneous emission decay curves from QDs, it is often implicitly assumed that the QDs decay through radiative recombination, while nonradiative processes are negligible. Unfortunately, this assumption is not generally valid, and omnipresent nonradiative processes must be considered. Only few experiments have addressed this issue. Robert et al. established an upper bound on the contribution from nonradiative processes of $25 \%$ by measuring the ratio of the biexciton to exciton emission intensity at saturation. ${ }^{12}$ Quantitative measurements of the radiative and nonradiative decay rates of QDs were only carried out recently using a modified LDOS both for colloidal QDs (Refs. 13 and 14) and for self-assembled QDs. ${ }^{15}$ Precise measurements of the radiative decay rates are essential since nanophotonic devices rely on the ability to manipulate the radiative processes, while nonradiative recombination leads to loss in the system.

As first pointed out by Purcell, ${ }^{16}$ the radiative decay rate of an emitter is modified inside a structured dielectric medium, which is due to the modification of the LDOS. In early experiments by Drexhage, ${ }^{17}$ this effect was experimentally demonstrated by positioning emitters in the proximity of a reflecting surface. Extending on this idea, we recently employed the modified LDOS near a semiconductor-air interface as a spectroscopic tool to extract radiative and nonradiative decay rates and from that infer the overlap between the electron and hole wave functions. ${ }^{15}$ This technique relies on the fact that the radiative decay rate is proportional to the LDOS, while the nonradiative decay rate is unaffected. In the present paper, we present additional data and compare the measured radiative decay rate to theory, which requires a detailed model of the QD electron and hole wave functions. We have measured the radiative decay rate at different emission energies, which reveals the dependence of the QD opti- 
cal properties on its size. We derive the Wigner-Weisskopf theory of spontaneous emission from QDs, predicting an exponential decay of the exciton population and the LDOS is derived for the applied interface geometry using the Green's function technique. We furthermore show that the radiative decay rate of a QD in a homogeneous medium is proportional to the square of the overlap integral between electron and hole wave functions and calculate the frequency dependence of this overlap using a simple two-band model of the QD. The QD model is discussed in details and compared to our experimental data employing realistic parameters as input to the theory. The pronounced size dependence of the electron-hole wave-function overlap is found to originate from the differences in effective mass and binding energy of the electron and hole. Furthermore, we investigate three different strain models for the QD and compare their predictions to experiment, thereby, providing valuable insight on the complex strain mechanisms of self-assembled QDs.

This paper is organized as follows. In Sec. II we present the experimental method and in Sec. III we present the experimental results. In Sec. IV we discuss the WignerWeisskopf model for spontaneous emission and derive the relation between the radiative decay rate, the LDOS, and the wave-function overlap. In Sec. V we combine the analytical expressions for the radiative rate with the numerical results for the wave-function overlap and compare theory to experiment. Finally, we present conclusions in Sec. VI.

\section{EXPERIMENTAL TECHNIQUE FOR DETERMINING THE RADIATIVE DECAY RATE OF QUANTUM DOTS}

Spontaneous emission of a photon from a QD occurs when an electron-hole pair (an exciton) recombines, as illustrated schematically in Fig. 1(a). As will be shown rigorously in Sec. IV, the QD radiative decay rate $\Gamma_{\text {rad }}\left(\mathbf{r}, \omega, \mathbf{e}_{\mathbf{p}}\right)$ in a structured environment is proportional to the projected LDOS $\rho\left(\mathbf{r}, \omega, \mathbf{e}_{\mathbf{p}}\right)$, where the projection is along the direction $\mathbf{e}_{\mathbf{p}}$ of the transition momentum matrix element, which corresponds to the orientation of the transition dipole moment. The LDOS is modified in an inhomogeneous dielectric medium due to optical reflections at interfaces. In emission experiments, the total decay rate is measured, which can be expressed as ${ }^{18}$

$$
\Gamma\left(\mathbf{r}, \omega, \mathbf{e}_{\mathbf{p}}\right)=\Gamma_{\operatorname{rad}}^{\mathrm{hom}}(\omega) \frac{\rho\left(\mathbf{r}, \omega, \mathbf{e}_{\mathbf{p}}\right)}{\rho_{\text {hom }}(\omega)}+\Gamma_{\mathrm{nrad}}(\omega),
$$

where $\rho_{\text {hom }}(\omega)$ is the density of optical states for a homogeneous medium, and $\Gamma_{\text {nrad }}(\omega)$ is the rate for nonradiative recombination. $\omega$ is the emission frequency and thus $\hbar \omega$ is the emission energy, and $\mathbf{r}$ is the position of the QD. Nonradiative recombination is due to intrinsic QD processes and thus independent of the LDOS. $\Gamma_{\mathrm{rad}}^{\mathrm{hom}}(\omega)$ is the QD radiative rate in a homogenous medium without any boundaries. In our case, the refractive index of the medium is $n=3.5$ corresponding to that of GaAs. Investigating $\Gamma_{\mathrm{rad}}^{\mathrm{hom}}(\omega)$ in detail provides valuable insight into the properties of the exciton wave function confined in the QD potential.

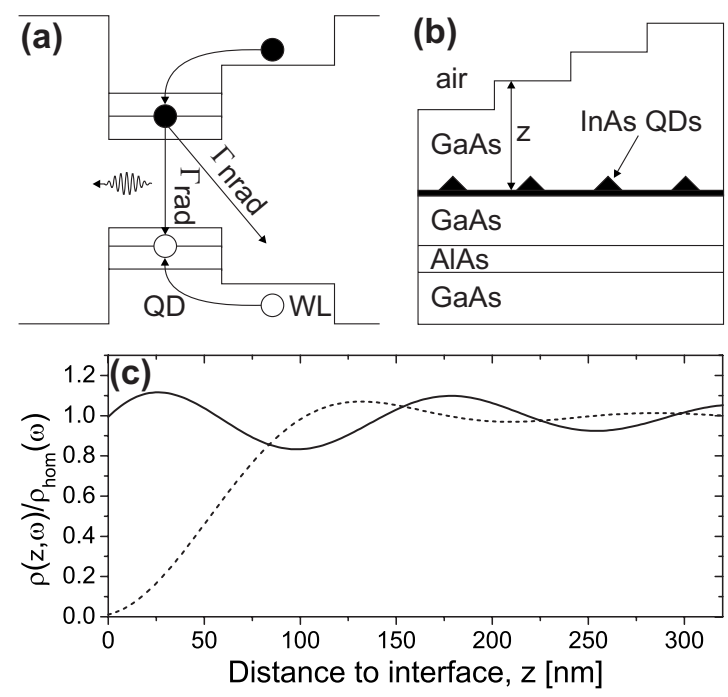

FIG. 1. (a) Schematic band diagram illustrating the spontaneous emission process in a QD. An electron is excited optically from a valence band to a conduction-band wetting-layer (WL) state and the generated electron and hole relax to the lowest-energy QD state on a picosecond time scale. The electron can subsequently decay by either radiative or nonradiative recombination with rates $\Gamma_{\text {rad }}$ and $\Gamma_{\text {nrad }}$, respectively. (b) Schematic illustration of the sample under investigation. InAs QDs are embedded in GaAs and positioned at different distances $z$ to the GaAs-air interface. (c) The LDOS as a function of distance $z$ to a GaAs-air interface for a dipole orientation parallel (solid curve) or perpendicular (dashed curve) to the interface.

The exact nature of nonradiative recombination in QDs is not yet fully understood. It is often implicitly assumed that nonradiative recombination is negligible, but as we will see in the following, even for very weak excitation intensities, this is not a valid assumption. Possible nonradiative processes include surface recombination at the interfaces between the QD and the surrounding semiconductor material, Auger processes, and trapping of electron and/or holes at defects, ${ }^{19}$ and any first-principles calculation of these effects is a tremendous task. Reliable ways of extracting the radiative and nonradiative parts of the decay rate are therefore essential

The radiative and nonradiative decay rates can be separated by time-resolved spontaneous emission measurements if the QDs are placed in an environment with a known LDOS [cf. Eq. (1)]. A planar interface between two regions with different refractive indices is the most simple example of an inhomogeneous dielectric medium. ${ }^{20}$ For this particular geometry, the LDOS can be calculated exactly. Here we employ the interface between GaAs $(n=3.5)$ and air $(n=1)$ as illustrated in Fig. 1(b). We calculate the LDOS by the Green's function technique and the results for dipole orientations parallel or perpendicular to the interface are shown in Fig. 1(c). We stress that no assumptions need to be made about, e.g., the QD density and the excitation beam profile in order to employ this experimental technique, as opposed to alternative ways of determining the radiative decay rate such as by absorption spectroscopy. ${ }^{21-23}$ 

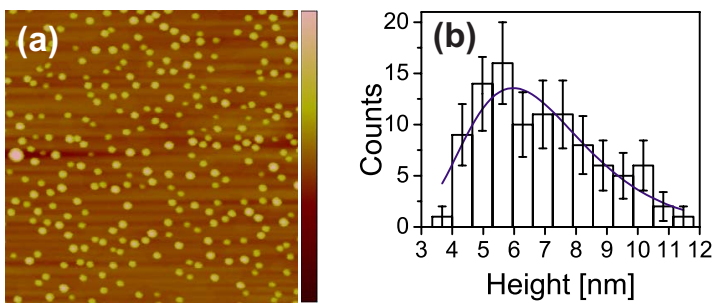

FIG. 2. (Color online) (a) Topographic atomic force micrograph depicting a surface area of $1 \times 1 \mu \mathrm{m}^{2}$ of uncapped QDs on the unprocessed wafer. The color scale runs from 0 to $20 \mathrm{~nm}$. (b) Histogram of the QD height measured by analysis of the AFM data. The blue line is a fit to the histogram data using a log-normal distribution as discussed in the text.

\section{MEASUREMENTS OF SPONTANEOUS EMISSION DECAY RATES NEAR A SEMICONDUCTOR-AIR INTERFACE}

The starting point of our investigations is a GaAs wafer grown by molecular-beam epitaxy (MBE). The QDs were grown using the Stranski-Krastranov method on a (001) GaAs substrate. The growth sequence was $50 \mathrm{~nm}$ AlAs, 610 $\mathrm{nm}$ GaAs, 2.0 monolayers (MLs) InAs, $300 \mathrm{~nm}$ GaAs cap, and finally 2.0 ML InAs. The AlAs layer was included for an optional epitaxial lift-off process, which was not employed here. Both InAs layers formed self-assembled QDs, but only the embedded layer was optically active because nonradiative surface recombination dominates for QDs at the surface. However, since the QDs at the surface were fabricated under identical growth conditions as the embedded ones, the density of the active QDs can be determined by atomic force microscopy (AFM) of the sample surface.

Such an atomic force micrograph is shown in Fig. 2(a). The QD height can be determined accurately from this measurement, but the measured width is convolved with the tip shape function. We used the AFM data in Fig. 2(a) to obtain a histogram of the heights recorded for 100 randomly selected QDs. The result is shown in Fig. 2(b). We fit the height histogram by a log-normal distribution given by $f(h)=\frac{h_{0}}{\sigma \sqrt{2 \pi} h} \exp \left(-\frac{(\ln (h)-\mu)^{2}}{2 \sigma^{2}}\right)$ with $h_{0}=68, \sigma=0.32$, and $\mu=1.9$, where $h$ is a dimensionless length scale normalized to $1 \mathrm{~nm}$. In Sec. V, we test the applicability of the height distribution for detailed quantum dot models.

From the AFM data, we found a QD density of $250 \mu \mathrm{m}^{-2}$, which corresponds to an average interdot distance of $60 \mathrm{~nm}$. This number should be compared to typical length scales for various relevant QD interactions. Carrier tunneling is negligible for distances beyond $15 \mathrm{~nm}$ (Ref. 24) and the dipoledipole interaction is only significant for distances close to the Förster radius, which is typically $2-9 \mathrm{~nm} .{ }^{18}$ Therefore, the measurements performed here provide ensemble averaged values of single QD properties with the advantage of an excellent signal-to-noise ratio in the measurements.

The wafer was processed by standard UV lithography and wet chemical etching in five subsequent steps with nominal etch depths of $160,80,40,20$, and $10 \mathrm{~nm}$ by which we obtained 32 fields with specific distances from the QDs to the semiconductor surface. The 32 fields were nominally

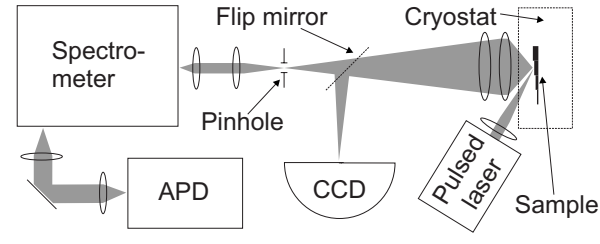

FIG. 3. Illustration of the optical measurement setup. The sample is kept at $14 \mathrm{~K}$ in a cryostat and illuminated by a pulsed laser. The spontaneously emitted light is collected and can be directed either to a charge-coupled device camera for sample alignment or to a spectrometer equipped with a fast single-photon counting avalanche photodiode (APD) for time-resolved measurements.

equidistantly spaced with $10 \mathrm{~nm}$ spacing. The wet etching was done using an etchant comprised of $\mathrm{H}_{3} \mathrm{PO}_{4}(85 \%), \mathrm{H}_{2} \mathrm{O}_{2}$ (30\%), and $\mathrm{H}_{2} \mathrm{O}$ in the ratio 3:1:60, which has an etch rate on GaAs at room temperature of $1 \mathrm{~nm} / \mathrm{s}$. We found that this etchant results in surfaces of good optical quality with low surface roughness. A schematic illustration of the resulting sample is shown in Fig. 1(b). Finally, we measured the actual distance from the QDs to the semiconductor surface by using a combination of secondary-ion mass spectroscopy and surface profiling from which we found typical depth uncertainties of $\pm 3.0 \mathrm{~nm}$.

The experimental setup is illustrated in Fig. 3. The sample was kept at $14 \mathrm{~K}$ and illuminated by a mode-locked Ti:sapphire laser emitting $300 \mathrm{fs}$ pulses at $1.45 \mathrm{eV}$, which corresponds to excitation of the wetting-layer states of the QD ensemble. The repetition rate was $80 \mathrm{MHz}$ and we used an excitation intensity of $7 \mathrm{~kW} / \mathrm{cm}^{2}$, which corresponds to less than 0.1 excitons per QD generated in the wetting layer per pulse, i.e., only the QD ground state is populated. Excitation of the WL states is advantageous since the same density of excitons is generated independent of sample thickness, which would not be the case for excitation in the GaAs barrier since the samples have different thicknesses. The pump configuration is illustrated in Fig. 1(a). The spontaneous emission from the QD ensemble was collected and then dispersed by a monochromator with a spectral resolution of 2.6 $\mathrm{meV}$ from which it was directed onto a fast silicon APD. The measured decay curves exhibit a biexponential decay evidenced by values of $\chi_{r}^{2}$ near unity for the biexponential fits for all distances. Further details on the analysis of the decay curves can be found in Ref. 15.

We measured the decay curves for 32 nominally equidistantly spaced distances to the interface. We found that for the two distances closest to the interface, there was no detectable spontaneous emission due to the very close proximity of the interface and/or damage by the etching process. In Fig. 4(a), we show the extracted fast and slow decay rates versus the LDOS normalized to the density of states of a homogeneous medium for the remaining 30 samples. The normalized LDOS is that of a dipole orientation parallel to the interface. The fast decay rate is expected to depend linearly on the normalized LDOS. However, close to the interface ( $z$ $\leq 75 \mathrm{~nm}$ ), the measured decay rate is found to be systematically larger than expected by theory. This deviation could be due to enhanced recombination rates induced by, e.g., scattering or impurities at the semiconductor surface. Another 


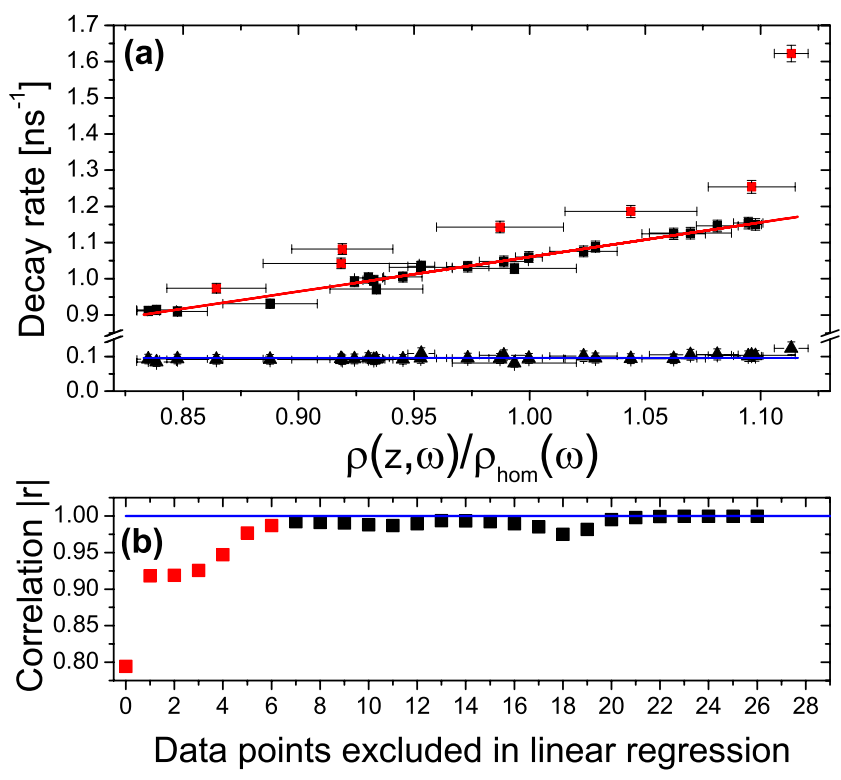

FIG. 4. (Color online) (a) Measured fast decay rate (black and red squares) at an emission energy of $1.204 \mathrm{eV}$ versus the normalized LDOS for a dipole orientation parallel to the semiconductor-air interface. The red line shows a linear fit, where only the black points have been included. The red points have been omitted because at positions close to the interface a systematic deviation from theory was observed, as quantified by the data in the lower panel. The slow decay rate (black triangles) is independent of the LDOS and is therefore dominated by nonradiative decay. The blue horizontal line shows the average nonradiative rate $\Gamma_{\text {nrad }}=0.096 \mathrm{~ns}^{-1}$. (b) Correlation between data and theory for the linear regression of the radiative rate when systematically excluding the points nearest the interface in the fit. The correlation parameter converges to a value close to unity (blue line) when seven points (marked in red) have been excluded.

mechanism which could cause deviations near the interface is changes in the overlap of electron and hole wave functions and hence changes in the radiative decay rate due to the quantum-confined Stark effect. This could arise from a built-in electric field due to the combined effect of Fermilevel pinning at the semiconductor surface and unintentional background impurity doping.

In order to exclude these effects in our analysis of intrinsic QD properties, we systematically excluded the data points closest to the interface in the fit. We found that the linear regression correlation parameter ${ }^{25}$ obtained from the fit in Fig. 4(a) saturated close to the ideal value of unity when excluding the seven innermost data points [see Fig. 4(b)]. These points were consequently abandoned in the analysis. For distances $z \geq 75 \mathrm{~nm}$, we find an excellent agreement between theory and experiment, which allows reliable extraction of the radiative and nonradiative rates of the QDs. The slow rate does not vary with the distance to the interface, so we conclude that it is dominated by nonradiative decay. It is attributed to the recombination of dark excitons and investigations of the dynamics will be published elsewhere. ${ }^{26}$ In the remainder of this paper, we will consider only the fast decay rate.

The linear fit in Fig. 4(a) is based on Eq. (1) and contains two free parameters, namely, the homogeneous radiative de-

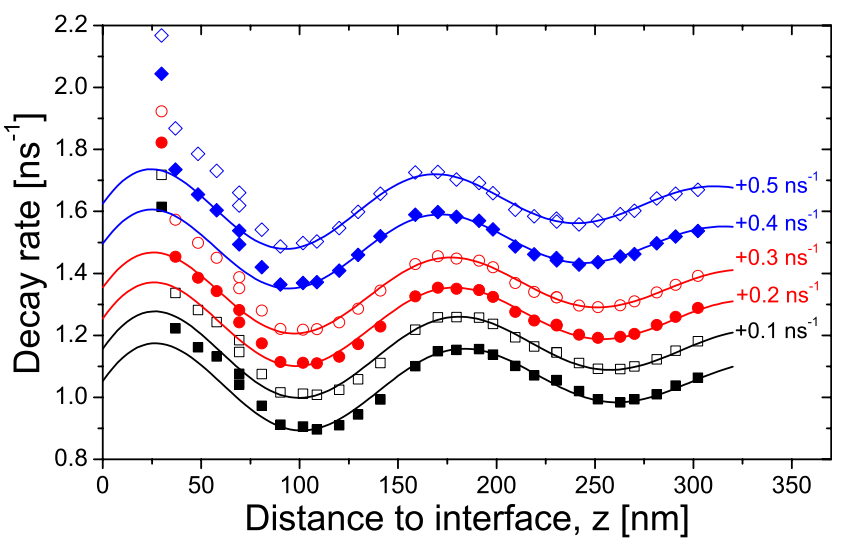

FIG. 5. (Color online) Decay rates as a function of distance to the interface for six different emission energies. Each curve has been vertically offset by $0.1 \mathrm{~ns}^{-1}$ for visual clarity. The fits have been obtained using a systematic exclusion of data points near the interface [cf. Fig. 4(b)]. The emission energies are $1.170 \mathrm{eV}$ (solid black squares), $1.187 \mathrm{eV}$ (open black squares), $1.204 \mathrm{eV}$ (solid red circles), $1.216 \mathrm{eV}$ (open red circles), $1.252 \mathrm{eV}$ (solid blue diamonds), and $1.272 \mathrm{eV}$ (open blue diamonds). For all emission energies, we note the excellent agreement with theory for $z>75 \mathrm{~nm}$.

cay rate $\Gamma_{\text {rad }}^{\text {hom }}$ and the nonradiative decay rate $\Gamma_{\text {nrad }}$. We obtain $\Gamma_{\mathrm{rad}}^{\mathrm{hom}}=0.95 \pm 0.03 \mathrm{~ns}^{-1}$ and $\Gamma_{\mathrm{nrad}}=0.11 \pm 0.03 \mathrm{~ns}^{-1}$ at an emission energy of $1.204 \mathrm{eV}$. The excellent agreement between experiment and theory confirms that the QD dipole moment is oriented perpendicular to the growth direction, which was previously established by absorption measurements. ${ }^{27}$

We measured the decay curves at six energies across the inhomogeneously broadened emission spectrum. When extracting the fast rate from the fits, we obtain the curves shown in Fig. 5. The different emission frequencies shift the curves along the abscissa and more importantly the amplitude and ordinate offsets are changing, which corresponds to changes in $\Gamma_{\text {rad }}^{\text {hom }}$ and $\Gamma_{\text {nrad. }}$ This shows directly the frequency dependence of $\Gamma_{\mathrm{rad}}^{\mathrm{hom}}$ and $\Gamma_{\text {nrad }}$, which will be discussed below.

The total decay rate in a homogeneous medium was extracted from Fig. 5 by the method outlined above and the result is shown in Fig. 6. The total decay rate increases with increasing emission energy, which could suggest that the radiative rate increases with energy, as has been reported for colloidal QDs. ${ }^{28}$ However, the opposite turns out to be true for self-assembled QDs. In this case, $\Gamma_{\mathrm{rad}}^{\mathrm{hom}}(\omega)$ is found to decrease with increasing energy, and the overall increase in the total rate is due to the pronounced increase in $\Gamma_{\text {nrad }}(\omega)$ with emission energy. It should be stressed that such variations in the radiative rate can be assessed only because a modified LDOS is employed allowing to separate radiative and nonradiative contributions. The striking energy dependence of the radiative rate can be explained as being due to the dependence of the electron and hole wave functions on the size of the QD, which will be discussed and analyzed in detail in Sec. V.

At low excitation power, nonradiative recombination may be due to thermally activated carrier escape or trapping at the QD-GaAs interface. ${ }^{29,30}$ Our measurements of the energy de- 


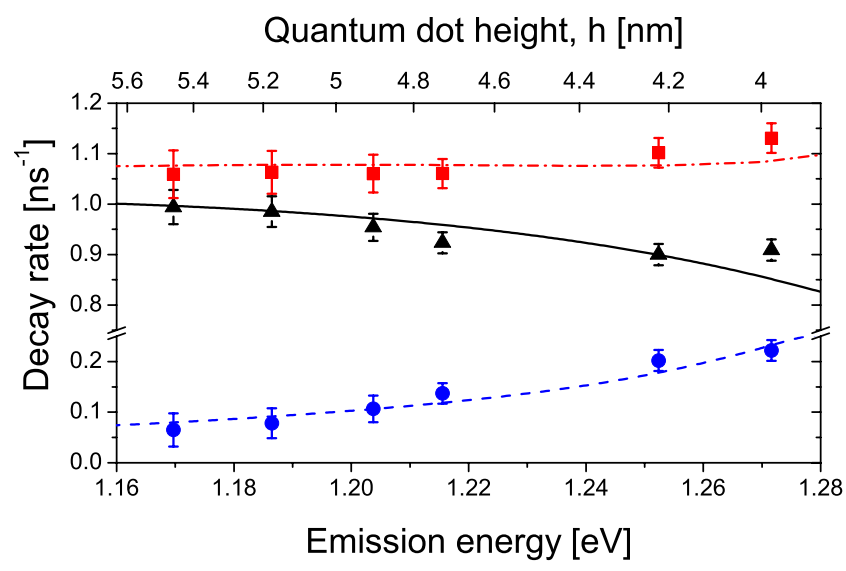

FIG. 6. (Color online) The total decay rate (red squares), radiative decay rate (black triangles), and nonradiative decay rate (blue circles) as a function of QD emission energy. The radiative rate decreases with energy but the nonradiative rate simultaneously increases so that the total (measured) decay rate increases with increasing energy. The solid black line is the result of the theoretical model of the radiative decay rate using an aspect ratio of $1 / 2$, omitting the wetting layer, and for an indium mole fraction of 0.95 . The dashed blue curve shows the theoretical model of the nonradiative decay. The dash-dotted red line shows the total decay rate given by the sum of the two theoretical curves. The top scale shows the heights used in the calculations. The details of the calculation of the nonradiative rate are discussed in the text and the calculation of the radiative rate is discussed in Sec. V.

pendence of the nonradiative rate enable a test of these models. Although the nanoscopic details of these processes are very complex, the energy dependence can be derived from simple scaling arguments. We assume that nonradiative recombination due to thermally activated carrier escape is of the form $\Gamma_{\text {nrad }}(\omega) \propto \exp \left\{-\left[E_{b}-E(\omega)\right] / k_{B} T\right\}$, where $E(\omega)$ is a function describing the energy of the system, i.e., the electron or hole quantization energy, $E_{b}$ is the barrier height, e.g., the electron or hole confinement potential or an impurity potential, and $k_{B}$ is Boltzmann's constant. The energy span of the experimental data in Fig. 6 is $100 \mathrm{meV}$, which means that $E(\omega)$ varies with approximately $50 \mathrm{meV}$ for each band. This is much larger than $k_{B} T=1.2 \mathrm{meV}$ at $14 \mathrm{~K}$ so the thermally excited carrier escape would lead to a much stronger energy dependence than observed and we can rule out this mechanism. For recombination at crystal defects at the QD-GaAs interface, the recombination scales with the surface-tovolume ratio, i.e., $\Gamma_{\text {nrad }}(\omega) \propto 1 / h(\omega)$, where $h(\omega)$ is the radius of the QD and we have assumed an aspect ratio of $1 / 2$. Here we define the aspect ratio as the ratio of height to width of the QD. We can only model our nonradiative decay data by subtracting an offset $h_{0}$ from the heights used in the calculations of the radiative rate. This could indicate corrections to the simple scaling relation due to the finite thickness of the QD-GaAs interface due to intermixing or discrepancies between the heights used in our model and the actual confinement potential. In Fig. 6, we show the result of this model, where $\Gamma_{\text {nrad }}(\omega)=v /\left[h(\omega)-h_{0}\right]$ and the QD heights are shown on the upper axis. Here the surface recombination constant is $v=0.18 \mathrm{~m} / \mathrm{s}$ and $h_{0}=3.2 \mathrm{~nm}$. This model shows a good

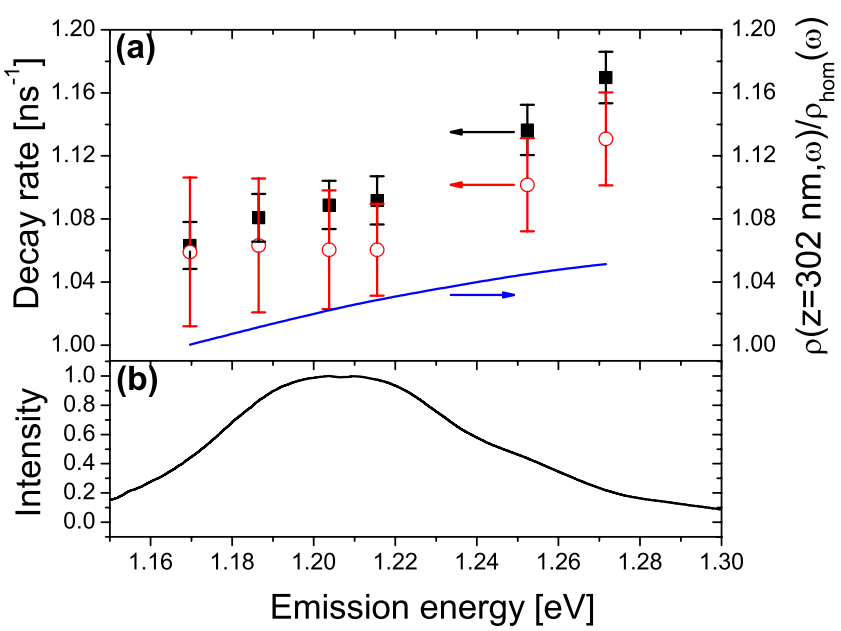

FIG. 7. (Color online) (a) The fast decay rate obtained from measurements on the unprocessed wafer (solid black squares) and the total decay rate in a homogenous medium (open red circles) obtained using the rigorous separation of radiative and nonradiative homogeneous decay rates. Evidently, the frequency dependence of the normalized LDOS (solid blue line) for the unprocessed wafer $(z=302 \mathrm{~nm})$ results in faster decay rates for QDs on the unprocessed wafer than would be the case in a homogeneous medium. (b) Normalized inhomogeneously broadened emission spectrum of the QDs obtained under weak excitation.

agreement with experiment, which supports that the nonradiative recombination in our QDs is due to recombination at defects at the QD-GaAs interface.

To conclude this section, we want to stress some potential pitfalls in the interpretation of the frequency dependence of spontaneous emission decay rates from QDs. As already pointed out, it is decisive to include the effects of nonradiative recombination and implement a technique that allows to separate it from radiative recombination. Thus, an a priori assumption of negligible nonradiative recombination would erroneously lead to the conclusion that the radiative rate increases with energy. Furthermore, when extracting quantitative data for the QD decay rates, one has to be aware of the influence of the presence of interfaces. In a homogeneous medium, the LDOS is proportional to the emission energy squared, but this is not the case in proximity of interfaces. In Fig. 7(a), we compare the measured total decay rate versus emission energy for an unprocessed wafer and compare to the total rate that QDs in a homogeneous medium would exhibit. The latter has been obtained using the LDOS technique explained above and provides "undisturbed" QD properties. For the unprocessed wafer, the distance to the interface is $302 \mathrm{~nm}$, and for this distance the LDOS increases with increasing energy [cf. the solid blue line in Fig. 7(a)]. This frequency dependence of the LDOS modifies the measured emission rates and is an example of the importance of considering nearby interfaces in quantitative assessment of QD properties. This implies that a homogenous medium cannot be approximated by an unprocessed wafer unless a very thick capping layer is grown on top of the QDs. In a recently published paper, ${ }^{31}$ the energy dependence of quantum dot lifetimes was investigated. The authors assumed a wavefunction overlap and quantum efficiency of unity and found 
that the measured decay rates were generally faster than expected from theory. This led the authors to conclude that the strong confinement model of QDs fails. We find on the contrary that the strong confinement model is fully valid for QDs provided that the frequency dependence of the LDOS is taken properly into account and that the quantum efficiency and wave-function overlap are measured.

\section{THEORETICAL DESCRIPTION OF SPONTANEOUS EMISSION FROM QUANTUM DOTS DUE TO INTERACTION WITH THE QUANTIZED ELECTROMAGNETIC FIELD}

In this section, we give a theoretical description of the radiative decay of excitons in QDs. QDs are many-particle objects and the quantum states of QDs is properly described in a many-particle picture. However, here we will restrict to a single-particle approximation because it is known to give correct results while being mathematically simpler. ${ }^{19,29}$ For sufficiently small QDs, the energy difference between bound states in the QDs is much larger than the Coulomb energy and the effect of the Coulomb interaction on the internal exciton dynamics becomes negligible. ${ }^{32}$ This means that the electron and hole forming the exciton may be considered independent, which is the strong confinement model. Furthermore, we employ here a two-band description of the QD including the effects of a wetting layer and strain, which is sufficient to capture the essential properties of QDs. ${ }^{33,34}$ The envelope function approach we use here is valid even for QD sizes as small as an atomic unit cell because the small effective masses and shallow confinement potentials in this system ensure that the envelope functions are slowly varying on the atomic scale even if the confinement potential is not. ${ }^{35}$

The inclusion of more bands is important for studying excited states of quantum dots, but for ground-state transitions the effects are small. It has been estimated that the inclusion of more bands in calculations of ground-state energies is relevant only when the lateral quantum dot dimensions are known within $\pm 1.5 \mathrm{~nm} .{ }^{36}$ In present experiments, the size of the confinement potential of the quantum dots is not known to such an accuracy and therefore a two-band model is sufficient. Also the inhomogeneity of the strain field in real structures can be used for detailed comparisons between experiment and theory only if the nanoscopic atomic configuration of the QDs is known with a very high accuracy. Therefore, we investigate homogeneous strain models, which have the additional benefit that they limit the number of free parameters in our models. These assumptions are supported $a$ posteriori by the fact that we are able to model our experimental data very well.

Our objective here is to explore the validity of the twoband model by a thorough comparison to our measurements of the radiative decay rate and the emission spectrum, which is carried out in Sec. V. Thorough explorations of simple QD models are much needed since QD models, which are complete and correct at the atomic scale, are outside reach both due to the lack of experimental knowledge about exact atomic composition and computational complexity.

Spontaneous emission occurs due to the interaction of the exciton with a continuum of vacuum radiation modes. A rig-

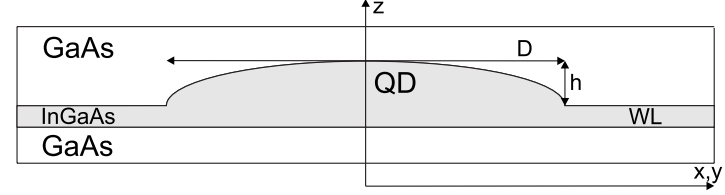

FIG. 8. Schematic illustration of the QD geometry used to calculate the envelope wave functions. We consider a lens-shaped QD with lateral base diameter $D$ and height $h$ consisting of $\operatorname{In}_{c} \mathrm{Ga}_{1-c} \mathrm{As}$ on a WL. The symmetry axis $z$ is indicated along with the radial directions $x, y$.

orous description of spontaneous emission requires a fully quantum description, where both the radiation field and the exciton states in the QD are quantized. We employ here the Wigner-Weisskopf model of spontaneous emission, which is valid when the LDOS varies slowly with frequency over the linewidth of the QD. This is an excellent approximation for the dielectric structures investigated here but may break down for QDs in photonic crystal leading to intricate nonMarkovian dynamics. ${ }^{37}$ The radiative coupling strength is determined by the electron momentum matrix element and depends furthermore sensitively on the overlap between the electron and hole envelope wave functions that in turn gives rise to the frequency dependence of the radiative decay rate. We derive here this frequency dependence, which will be compared in detail to the experimental data in Sec. V.

\section{A. Wigner-Weisskopf theory of spontaneous emission from quantum dots}

According to effective-mass theory, the solution to the Schrödinger equation for an electron in a solid is given by $\Psi_{n}(\mathbf{r})=F_{n}(\mathbf{r}) u_{n, \mathbf{0}}(\mathbf{r})$, where $F_{n}(\mathbf{r})$ is the envelope function, $u_{n, \mathbf{0}}(\mathbf{r})$ is the Bloch function evaluated at the band edge $\mathbf{q}$ $=\mathbf{0}$, and $n \in\{c, v\}$ denotes the conduction $(c)$ or valence $(v)$ band. The envelope function is the solution to the effectivemass Schrödinger-like equation governed by the Hamiltonian $^{38}$

$$
H_{0}(\mathbf{r})=H_{\mathrm{kin}, n}+V_{n}(\mathbf{r}),
$$

where $H_{\mathrm{kin}, n}$ is the kinetic-energy operator, and $V_{n}(\mathbf{r})$ is the band confinement potential. Here we consider lens-shaped QD geometries as shown in Fig. 8. For the conduction band, the effective mass is isotropic, so that $H_{\mathrm{kin}}=-\frac{\hbar^{2}}{2 m_{0}} \nabla \cdot \frac{1}{m_{e}(\mathbf{r})} \nabla$, where $m_{0}$ is the elementary electron mass and $m_{n}(\mathbf{r})$ is the effective mass. For the valence band, the anisotropy of the effective mass must be taken into account, which is discussed in Appendixes A and B.

For the valence band $m_{v}<0$ and $V_{v}(\mathbf{r})<0$, which leads to negative eigenenergies. In the electron-hole representation, ${ }^{39}$ we define the hole in the valence band as a particle with positive effective mass $m_{h}(\mathbf{r})=-m_{v}(\mathbf{r})$ subject to a positive confinement potential $V_{h}(\mathbf{r})=-V_{v}(\mathbf{r})$ yielding positive eigenenergies. Clearly, the envelope function remains the same in the new representation, i.e., $F_{h}(\mathbf{r})=F_{v}(\mathbf{r})$. For III-V semiconductors, the valence band is comprised of degenerate bands with different effective masses. However, for QDs strain lifts this degeneracy and we may consider the valence 
band as a single band (the heavy-hole band). We discuss details of the band structure in the presence of strain in Appendix A.

We describe the light-matter interaction by the Hamiltonian $H^{\prime}(\mathbf{r}, t)=-\frac{q}{m_{0}} \mathbf{p} \cdot \mathbf{A}(\mathbf{r}, t)$, where $q$ is the elementary charge, $\mathbf{p}$ is the momentum operator, and $\mathbf{A}(\mathbf{r}, t)$ is the vector potential of the quantized electromagnetic field. The latter is given by ${ }^{40,41}$

$$
\mathbf{A}(\mathbf{r}, t)=\sum_{\mu} \frac{\epsilon_{\mu}}{\omega_{\mu}} \hat{\mathbf{e}}_{\mu}\left[A_{\mu}(\mathbf{r}) a_{\mu} e^{-i \omega_{\mu} t}+A_{\mu}^{*}(\mathbf{r}) a_{\mu}^{\dagger} e^{i \omega_{\mu} t}\right]
$$

Here $\mu=(\mathbf{k}, s)$ is the combined wave vector $\mathbf{k}$ and polarization index $s \in\{1,2\}, \omega_{\mu}$ is the optical angular frequency, $\epsilon_{\mu}=\sqrt{\frac{\hbar \omega_{\mu}}{2 \epsilon_{0}}}$ is a normalization constant with $\epsilon_{0}$ denoting the vacuum permittivity, $\hat{\mathbf{e}}_{\mu}$ is a unit vector in the direction of the polarization $s, A_{\mu}(\mathbf{r})$ is the spatial field distribution function, and $a_{\mu}$ and $a_{\mu}^{\dagger}$ are the field annihilation and creation operators, respectively. In a homogeneous medium, the field distribution functions are given by plane waves $A_{\mu}(\mathbf{r})=\frac{e^{i \mathbf{k} \cdot \mathbf{r}}}{\sqrt{\epsilon_{\mathrm{r} V} V}}$, where $\epsilon_{r}=n^{2}$ is the static dielectric permittivity of the material. We are working in the Coulomb gauge in which the scalar potential of the electromagnetic field is zero and the divergence of the vector potential vanishes.

We consider the initial state $|i\rangle=|c\rangle \otimes|0\rangle$ with $|c\rangle$ $=F_{e}(\mathbf{r})\left|u_{c, 0}\right\rangle$, corresponding to a $\mathrm{QD}$ with one electron promoted to the conduction band and no photons in the radiation field. The final state relevant for spontaneous emission is $\left|f_{\mu}\right\rangle=|v\rangle \otimes\left|1_{\mu}\right\rangle$ with $|v\rangle=F_{h}(\mathbf{r})\left|u_{v, 0}\right\rangle$, where a photon is radiated to the mode $\mu$ while the excited electron has decayed to the valence band. In a two-band model, the states $|c\rangle$ and $|v\rangle$ form a complete set so that we can expand the interaction Hamiltonian by insertion of complete sets to obtain an expression containing the raising and lowering operators of the electronic system, which we define as $\sigma_{+}=|c\rangle\langle v|$ and $\sigma_{-}$ $=|v\rangle\langle c|$, respectively. It is convenient to change to the interaction picture, in which the time evolution of the raising and lowering operators are given by $\widetilde{\sigma}_{+}(t)=\sigma_{+} e^{i \omega_{0} t}$ and $\widetilde{\sigma}_{-}(t)$ $=\sigma_{-} e^{-i \omega_{0} t}$, where we have introduced the energy of the exciton transition $\hbar \omega_{0}$. Furthermore, assuming that the spatial distribution functions of the vector potential are slowly varying on the scale of the wave functions they can be evaluated at the center position $\mathbf{r}_{0}$ of the QD, which is the dipole approximation. The interaction Hamiltonian in the interaction picture now reads as

$$
\begin{aligned}
H^{\prime}\left(\mathbf{r}_{0}, t\right)= & -\frac{q}{m_{0}}\langle c|\mathbf{p}| v\rangle \sigma_{+} \cdot \sum_{\mu} \frac{\boldsymbol{\epsilon}_{\mu}}{\omega_{\mu}} \hat{\mathbf{e}}_{\mu} A_{\mu}\left(\mathbf{r}_{0}\right) a_{\mu} e^{-i \Delta_{\mu} t} \\
& -\frac{q}{m_{0}}\langle v|\mathbf{p}| c\rangle \sigma_{-} \cdot \sum_{\mu} \frac{\boldsymbol{\epsilon}_{\mu}}{\omega_{\mu}} \hat{\mathbf{e}}_{\mu} A_{\mu}^{*}\left(\mathbf{r}_{0}\right) a_{\mu}^{\dagger} e^{i \Delta_{\mu} t},
\end{aligned}
$$

where $\Delta_{\mu}=\omega_{\mu}-\omega_{0}$ and we have omitted two terms proportional to $e^{ \pm i \Delta_{\mu} t}$ since they are rapidly oscillating as a function of time, which is the rotating wave approximation.

The general state vector of the system can be expanded as

$$
|\Psi(t)\rangle=c_{e}(t)|i\rangle+\sum_{\mu} c_{\mu}(t)\left|f_{\mu}\right\rangle,
$$

where $c_{e}(0)=1$ and $c_{\mu}(0)=0$. By insertion into the Schrödinger equation in the interaction picture, we obtain the following equation of motion: ${ }^{40}$

$$
\begin{aligned}
\frac{d}{d t} c_{e}(t)= & -\frac{q^{2}}{2 \hbar m_{0}^{2} \epsilon_{0}}|\langle v|\mathbf{p}| c\rangle|^{2} \int_{0}^{t} d t^{\prime} c_{e}\left(t^{\prime}\right) \int_{-\infty}^{\infty} d \omega \frac{\rho\left(\mathbf{r}_{0}, \omega, \hat{\mathbf{e}}_{\mathbf{p}}\right)}{\omega} \\
& \times e^{-i\left(\omega-\omega_{0}\right)\left(t-t^{\prime}\right)},
\end{aligned}
$$

where we have included an integration over a Dirac delta function in frequency. $\rho\left(\mathbf{r}_{0}, \omega, \hat{\mathbf{e}}_{\mathbf{p}}\right)$ is the projected LDOS defined as

$$
\rho\left(\mathbf{r}_{0}, \omega, \hat{\mathbf{e}}_{\mathbf{p}}\right)=\sum_{\mu}\left|\hat{\mathbf{e}}_{\mathbf{p}} \cdot \hat{\mathbf{e}}_{\mu}\right|^{2}\left|A_{\mu}\left(\mathbf{r}_{0}\right)\right|^{2} \delta\left(\omega-\omega_{\mu}\right),
$$

where $\hat{\mathbf{e}}_{\mathbf{p}}$ is the unit vector specifying the direction of $\langle v|\mathbf{p}| c\rangle$. This direction is determined by the Bloch matrix element as discussed below. Since the term $\rho\left(\mathbf{r}_{0}, \omega, \hat{\mathbf{e}}_{\mathbf{p}}\right) / \omega$ in Eq. (6) varies on the scale of the emission energy $(\approx 1 \mathrm{eV})$, it is slowly varying on the scale of the homogeneous QD linewidth $(\approx 10 \mu \mathrm{eV})$. Thus, $\rho\left(\mathbf{r}_{0}, \omega, \hat{\mathbf{e}}_{\mathbf{p}}\right) / \omega$ can be evaluated at the emission frequency $\omega_{0}$ and taken outside the integral. In this case, the QD population decays exponentially $\left|c_{e}(t)\right|^{2}$ $=e^{-\Gamma_{\mathrm{rad}}\left(\mathbf{r}_{0}, \omega_{0}, \hat{\mathbf{e}}_{\mathbf{p}}\right) t}$ with the radiative decay rate given by

$$
\Gamma_{\mathrm{rad}}\left(\mathbf{r}_{0}, \omega_{0}, \hat{\mathbf{e}}_{\mathbf{p}}\right)=\frac{\pi q^{2}}{\hbar m_{0}^{2} \epsilon_{0}}|\langle v|\mathbf{p}| c\rangle|^{2} \frac{\rho\left(\mathbf{r}_{0}, \omega_{0}, \hat{\mathbf{e}}_{\mathbf{p}}\right)}{\omega_{0}} .
$$

This is the Wigner-Weisskopf result for spontaneous emission from solid-state emitters. It states that the radiative decay rate is proportional to the projected LDOS and the momentum matrix element. In the following subsection, we discuss the evaluation of these two terms. In the experiment, the number of photons emitted per time is measured, which is given by

$$
N(t)=\alpha \Gamma_{\mathrm{rad}} e^{-\left(\Gamma_{\mathrm{rad}}+\Gamma_{\mathrm{nrad}}\right) t},
$$

where additionally the rate for nonradiative recombination has been added, and $\alpha$ is an overall scaling parameter determined by the detection efficiency and the total number of photons recorded during the measurement period.

\section{B. Evaluation of the projected LDOS and the transition matrix element}

The projected LDOS can be calculated using the Green's function technique. In terms of the Green's tensor $\mathbf{G}\left(\mathbf{r}, \mathbf{r}^{\prime}, \omega\right)$, the projected LDOS is given by ${ }^{18,42}$

$$
\rho\left(\mathbf{r}, \omega, \hat{\mathbf{e}}_{\mathbf{p}}\right)=\frac{2 \omega}{\pi c_{0}^{2}}\left\{\hat{\mathbf{e}}_{\mathbf{p}} \cdot \operatorname{Im}[\mathbf{G}(\mathbf{r}, \mathbf{r}, \omega)] \cdot \hat{\mathbf{e}}_{\mathbf{p}}\right\},
$$

where $c_{0}$ is the speed of light in vacuum. The LDOS is a classical electromagnetic quantity obtained by solving Maxwell's equations. However, it enters the quantum-optical theory of light-matter interaction, where it describes the local density of vacuum modes that spontaneous emission can occur to. For the particular case of a semiconductor-air inter- 
face as considered here, the Green's tensor is obtained by solving the following closed expression: ${ }^{18,43}$

$$
\mathbf{G}(\mathbf{r}, \mathbf{r}, \omega)=\frac{i}{8 \pi k^{2}} \int_{0}^{\infty} d k_{\rho} \frac{k_{\rho}}{k_{z}}\left(\mathbf{M}_{0}+\mathbf{M}_{r}\right),
$$

where

$$
\mathbf{M}_{0}=\left[\begin{array}{ccc}
2 k^{2}-k_{\rho}^{2} & 0 & 0 \\
0 & 2 k^{2}-k_{\rho}^{2} & 0 \\
0 & 0 & 2\left(k^{2}-k_{z}^{2}\right)
\end{array}\right]
$$

and

$$
\mathbf{M}_{r}=\left[\begin{array}{ccc}
\left(k^{2} r^{s}-k_{z} r^{p}\right) & 0 & 0 \\
0 & \left(k^{2} r^{s}-k_{z}^{2} r^{p}\right) & 0 \\
0 & 0 & \left(2 k_{\rho}^{2} r^{p}\right)
\end{array}\right] e^{2 i k_{z} z}
$$

Here $k=|\mathbf{k}|$, where $\mathbf{k}=\left(k_{\rho}, k_{z}, k_{\phi}\right)$ is the $\mathbf{k}$ vector in cylindrical coordinates, $z$ is the distance from the QD to the interface, and $r^{s}\left(r^{p}\right)$ is the Fresnel reflection coefficient for $s$-polarized ( $p$-polarized) light. ${ }^{18}$ The result of the calculation for a GaAs-air interface is shown in Fig. 1(c).

We now consider the transition matrix element. Using the fact that the momentum operator is a differential operator $(\mathbf{p}=-i \hbar \nabla)$, that the envelope functions are slowly varying on the scale of a lattice parameter, ${ }^{29}$ and that the Bloch functions $u_{c / v, 0}(\mathbf{r})$ are orthogonal, which we will describe below, we obtain

$$
|\langle v|\mathbf{p}| c\rangle|^{2} \approx\left|\left\langle F_{h} \mid F_{e}\right\rangle\right|^{2}\left|\left\langle u_{v, 0}|\mathbf{p}| u_{c, 0}\right\rangle\right|^{2} \equiv\left|\left\langle F_{h} \mid F_{e}\right\rangle\right|^{2} \times \frac{m_{0} E_{p}(c)}{2} .
$$

This important result states that the transition matrix element is given by the product of the electron and hole wavefunction overlap and the squared Bloch matrix element $\left|\left\langle u_{v, 0}|\mathbf{p}| u_{c, 0}\right\rangle\right|^{2}$. The magnitude of the Bloch matrix element is a material parameter that is expressed in terms of the Kane energy $E_{p}(c) .^{29} c$ is the indium mole fraction in the $\mathrm{In}_{c} \mathrm{Ga}_{1-c} \mathrm{As}$ alloy.

In the Kane model, ${ }^{19,44}$ the valence-band Bloch functions are written as linear combinations of the basis functions $\left|u_{x}\right\rangle$, $\left|u_{y}\right\rangle$, and $\left|u_{z}\right\rangle$ that carry the symmetry properties of $p$ orbitals. The specific linear combination depends on the $\mathbf{q}$ vector of the envelope function. QDs grown by the Stranski-Krastanov technique are typically flat structures placed on top of a wetting layer, and quantization along the growth direction $(z)$ is therefore dominating. ${ }^{45}$ As a consequence, we can set $q_{x}$ $=q_{y}=0$, which is exact in the limit of a quantum well, leading to $\mathbf{q}=|\mathbf{q}| \hat{\mathbf{e}}_{z}$. In this case, the heavy-hole Bloch functions can be written as $\left|u_{v, 0}\right\rangle=\left|u_{h h}\right\rangle=\frac{1}{\sqrt{2}}\left(\left|u_{x}\right\rangle \pm i\left|u_{y}\right\rangle\right)$. The conduction-band Bloch functions have $s$ symmetry, so the Bloch functions for the valence and conduction bands are orthogonal as was used above. Therefore, the matrix element $\left|\left\langle u_{v, 0}|\mathbf{p}| u_{c, 0}\right\rangle\right|^{2}$ is nonzero only for $p_{x}$ and $p_{y}$ from which we conclude that the dipole axis of the QD for heavy-hole transitions is perpendicular to the growth direction in agreement with our experiment.

\section{Frequency dependence of the radiative decay rate of quantum dots}

We are now in a position to put together the results of the previous sections and calculate the frequency dependence of the spontaneous emission decay rates in a homogenous medium. By insertion of Eq. (7) in Eq. (8) and using the planewave expression for the field distribution functions, we readily obtain

$$
\Gamma_{\mathrm{rad}}^{\mathrm{hom}}(\omega)=\frac{\pi q^{2}}{\hbar m_{0}^{2} \boldsymbol{\epsilon}_{0}} \frac{1}{\epsilon_{r} V}|\langle v|\mathbf{p}| c\rangle|^{2} \frac{1}{\omega_{0}} \sum_{\mu}\left|\hat{\mathbf{e}}_{\mathbf{p}} \cdot \hat{\mathbf{e}}_{\mu}\right|^{2} \delta\left(\omega-\omega_{\mu}\right) .
$$

The sum over all optical modes $\mu$ can be converted to an integration over all $k$ vectors, where the dispersion relation for a homogeneous medium $\omega_{\mu}=k_{\mu} c_{0} / n$ is used. The sum over polarizations yields a factor of 2. Using also Eq. (14), we obtain the important relation

$$
\Gamma_{\mathrm{rad}}^{\mathrm{hom}}(\omega)=\frac{n q^{2}}{6 \pi \hbar m_{0} c_{0}^{3} \epsilon_{0}} E_{p}(c) \omega\left|\left\langle F_{h}(\omega) \mid F_{e}(\omega)\right\rangle\right|^{2} .
$$

Here we have indicated explicitly that the envelope wave functions depend on the emission energy since varying the QD size and, thereby, the emission energy, leads to modifications of the wave functions. This effect will be discussed in detail in Sec. V. Equation (16) is the key result used to interpret the experimental measurements of the radiative decay rate presented in this paper. It furthermore allows extracting an experimental value for the overlap between the electron and hole wave functions.

\section{COMPARISON BETWEEN EXPERIMENT AND THEORY FOR THE ELECTRON-HOLE WAVE-FUNCTION OVERLAP}

In this section, we calculate the electron-hole wavefunction overlap and the QD emission spectrum and compare to our experimental results. We investigate to what extent the QD heights obtained from AFM measurements of uncapped QDs [cf. Fig. 2] can be used as input to the models. Furthermore, we systematically test the model against experiment by varying parameters such as the indium mole fraction, the QD aspect ratio, the wetting-layer thickness, and the applied strain model within physically realistic boundaries. The model reproduces the decrease in the electron-hole wavefunction overlap with energy that we observed experimentally. However, our investigations lead to the conclusion that further knowledge of QD composition and more involved QD models would be required in order to reach full quantitative agreement between experiment and theory.

We describe the QD in the $(\rho, z)$ plane as one quarter of an ellipse with a fixed aspect ratio. Here $\rho$ denotes the radial direction in cylindrical coordinates. The QD geometry is solved numerically in a large simulation area, which spans $160 \mathrm{~nm}$ in the $\rho$ direction and $80 \mathrm{~nm}$ in the $z$ direction, ensuring that the proximity of the boundaries has no effect on the results. Further details on the numerical procedure are provided in Appendix B. We use the commercial finite ele- 
ment software package COMSOL with an adaptive mesh to solve the effective-mass equation [Eq. (B3)]. For each QD height, we obtain the transition energy and given the height distribution function measured by AFM (cf. Fig. 2), we calculate the emission spectrum.

The fact that we only observe transitions involving heavy holes motivates a further investigation of the analogy between QDs and quantum wells. In particular, the effect of strain on the electronic level structure is well understood for quantum wells. ${ }^{19}$ Strain has a significant effect on the level structure also for QDs but is often discussed in qualitative terms only due to the mathematical complexity and lack of experimental input on the exact QD geometry and composition. In the following, we model the QD strain similar to the case of a quantum well and compare the results to experiment. In a quantum well of InGaAs in GaAs, the compressive strain in the plane of the quantum well leads to an expansion in the direction perpendicular to the plane. This biaxial strain can be decomposed into a hydrostatic and a shear component. However, as opposed to the case of a quantum well, a QD cannot expand freely in the growth direction, which suggests that the hydrostatic component may dominate. Therefore, we compare three different strain models including: (1) hydrostatic and shear strain, (2) only hydrostatic strain, and (3) no strain. Strain modifies the band offsets and the valence-band effective masses, which is discussed in further detail in Appendix A.

Using the experimental data on both the QD height distribution function, the emission spectrum, and the frequency dependence of the wave-function overlap, we explore experimentally realistic parameters in order to match our experimental data. The first approach is to use the measured QD height distribution of Fig. 2 and include both shear and hydrostatic strain. Using optimized parameters corresponding to an aspect ratio of $1 / 6$ and an indium mole fraction of $39 \%$, we find a very good agreement with the emission spectrum, as shown in Fig. 9(a). This aspect ratio is surprisingly small and the electron-hole wave-function overlap extracted from the radiative decay rate offers a needed additional test of this parameter set. In Fig. 9(b), the experimentally determined wave-function overlap is plotted along with the resulting theory. ${ }^{46}$ The theory predicts correctly that the wave-function overlap decreases (increases) with emission energy (QD size), and the mechanism behind this effect is discussed in detail below. However, a systematic deviation between theory and experiment is observed, which turns out to be the case for all three strain models provided that the model parameters are constrained to optimally reproduce the measured spectrum. As we will discuss below, this deviation cannot be attributed to deficiencies in the theoretical model. We conclude from this result that the measured height distribution of uncapped QDs does not directly determine the actual confinement volume of the overgrown QDs that the experiments are performed on. This is because of complex redistribution and intermixing processes of indium and gallium, which will occur during growth and subsequent regrowth ${ }^{47,48}$ and these are likely to modify the QD confinement potential and strain significantly. Also, by comparing AFM measurements on uncapped QDs with transmission electron micrographs of capped QDs, it has been shown that QDs shrink by

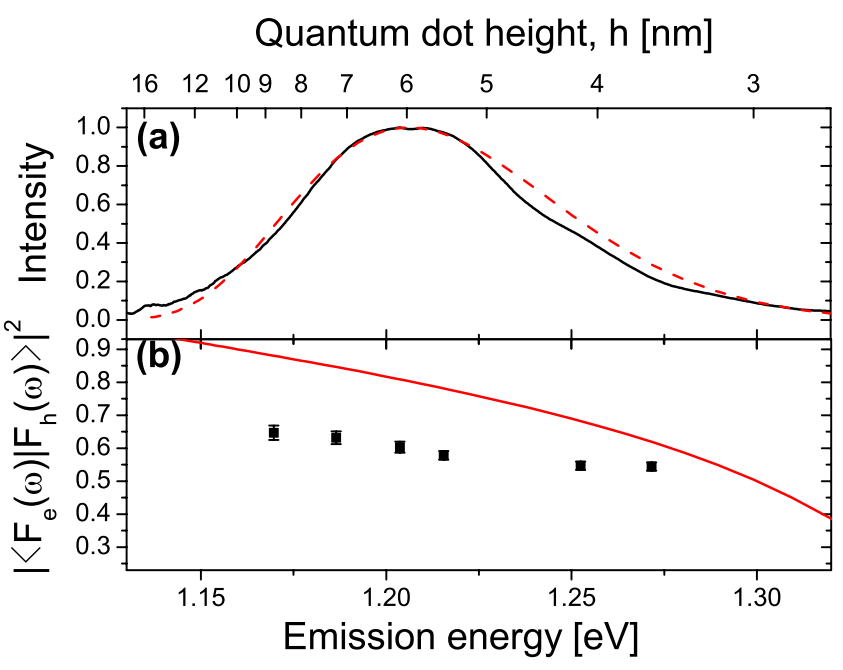

FIG. 9. (Color online) (a) Measured spontaneous emission spectrum (black curve, normalized) and calculated spectrum (dashed red curve) using the height distribution of the QDs from Fig. 2(b) and implementing strain model 1 . The resulting parameters have been optimized to fit the spectrum, and we find an aspect ratio of 1/6 and an indium mole fraction in the QD of 39\%. (b) Measured electronhole wave-function overlap (black squares) and the theoretical calculation (solid red line) using the same parameters as in (a).

overgrowth. ${ }^{49}$ Our results support the picture that gallium diffuses into the QDs during formation of QDs and partly diffuses out during the capping.

We have also investigated the impact of the lateral QD extension on the calculated spectrum and wave-function overlap within our model. It turns out that the lateral extension in has only quite small effects on the results. Changing the aspect ratio changes the quantum dot height axis relative to the emission energy axis (cf. Fig. 9) and hence the calculated spectrum but yields only very small changes in the frequency dependence of the wave-function overlap.

Thus, abandoning at this point the interpretation of the measured height distribution and its relation to the emission spectrum, we focus on the electron-hole wave-function overlap. Keeping the aspect ratio fixed at a realistic value of $1 / 2$ and excluding for the moment the wetting layer, we obtain the overlap shown in Fig. 10. Here the only free parameter is the mole fraction of indium $c$ in the QD and the different strain models are compared. Note that the experimentally determined wave-function overlap depends on $c$ since it enters through the Kane energy [cf. Eq. (16)]. We find that including both shear and hydrostatic strain leads to a disagreement between experiment and theory. This demonstrates that the strain model developed for quantum wells fails in the case of QDs, as opposed to what is sometimes assumed in the literature. ${ }^{38}$ Good agreement between experiment and theory can be obtained when including only hydrostatic strain or no strain at all for $c=0.95$ and $c=0.46$, respectively. Judging from experiments available in the literature, ${ }^{47,48}$ both these values of $c$ are reasonable for overgrown QDs, so there is no support for favoring one of the two surviving strain models. We are led to the conclusion that, in particular, shear strain is less significant for QDs compared to quantum wells, although further nanoscopic de- 


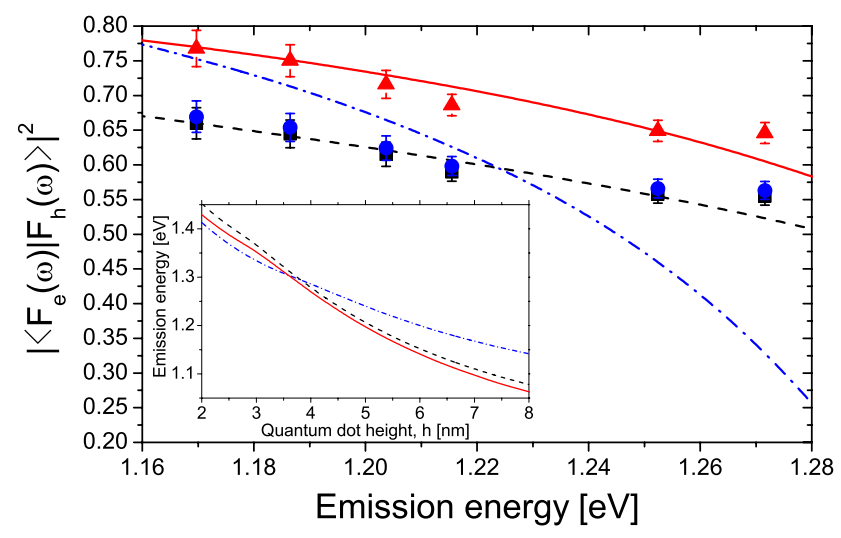

FIG. 10. (Color online) Calculated energy dependence of the squared electron and hole wave-function overlap using the three different strain models discussed in the text. The QD aspect ratio is $1 / 2$ and no wetting layer was included. The curves are calculated for strain model 1 with $c=0.51$ (dash-dotted blue line), strain model 2 with $c=0.95$ (solid red line), and strain model 3 with $c=0.46$ (dashed black line). The data points show the experimentally determined wave-function overlap for these three indium mole fractions using the same color coding. Clearly, strain model 1 does not describe the experimental data well, while both models 2 and 3 lead to very good agreement. The inset shows the dependence of the QD emission energy on height for the three different models using the same color coding as in the main figure.

tails of QD composition and geometry would be required for a further investigation of these issues.

Figure 11 investigates the effect of the wetting-layer thickness on the wave-function overlap. In this case strain is omitted, the aspect ratio is $1 / 2$, and the indium mole fraction is 0.46 . We find that for wetting-layer thicknesses below 4 ML, the wave-function overlap is only slightly modified in the emission energy range of interest to the experiment. For a very thick wetting layer (6 ML), the QD wave function is modified such that the monotonic decrease in the wavefunction overlap with energy observed for all other thicknesses does not apply. This behavior can be understood as follows: for very thick wetting layers and small QDs a significant part of both the electron and hole wave functions are

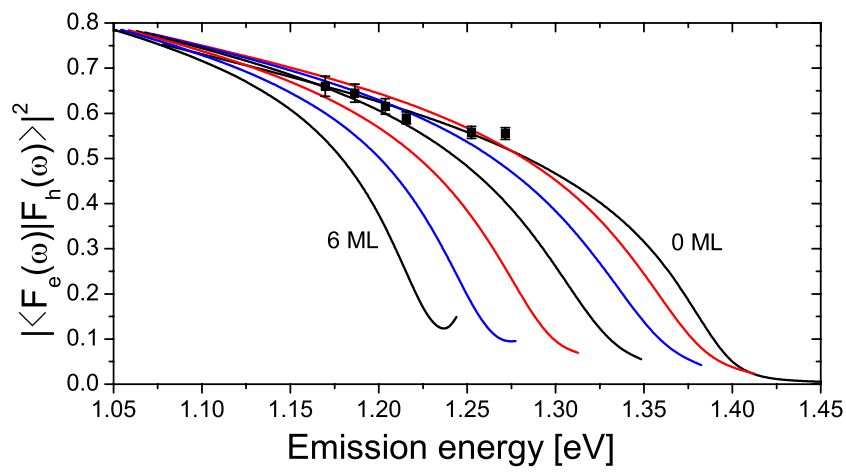

FIG. 11. (Color online) The effect of the thickness of the wetting layer on the calculated electron-hole wave-function overlap when varying between 0 and $6 \mathrm{ML}$ in steps of $1 \mathrm{ML}$. Here we have omitted strain and otherwise used the same parameters as in Fig. 10. The experimental data for $c=0.46$ are shown as black squares.

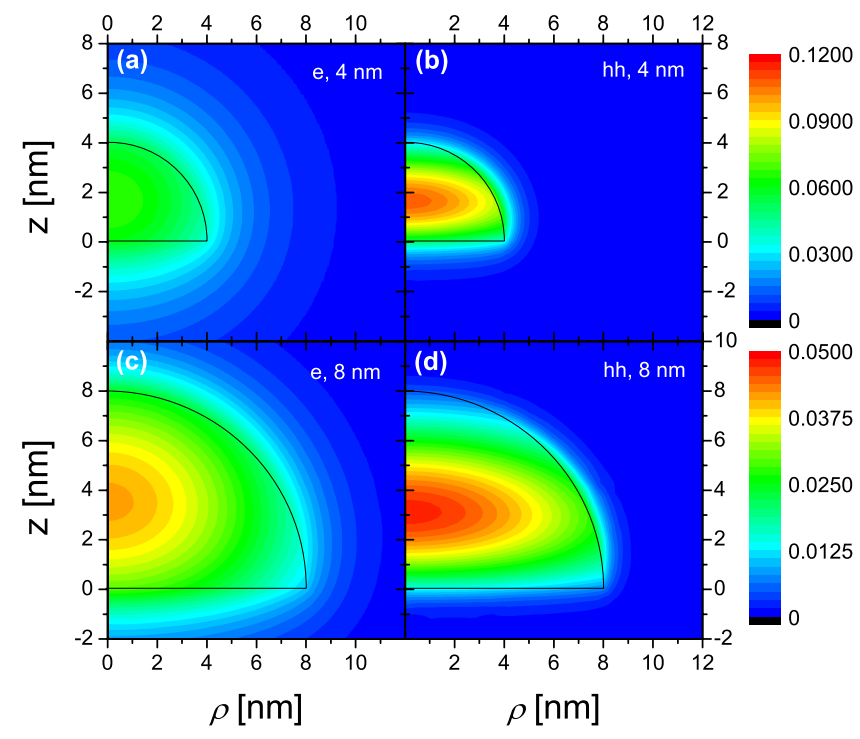

FIG. 12. (Color online) Contour plots in the radial plane of the amplitude of the wave function for electrons and holes. The parameters corresponding to the calculation for strain model 2 in Fig. 10 were used. (a) Electron wave function for a QD height of $4 \mathrm{~nm}$. (b) Hole wave function for a QD height of $4 \mathrm{~nm}$. (c) Electron wave function for a QD height of $8 \mathrm{~nm}$. (d) Hole wave function for a QD height of $8 \mathrm{~nm}$.

expelled from the QD giving rise to quantum well-like wetting-layer states that can have an increased mutual overlap. For the QD sample of the experiment, the wetting layer was on the order of 2 ML.

The above discussions point to a number of subtleties associated with a quantitative comparison between experiment and theory. These are mainly related to lack of knowledge about intrinsic properties of the QDs. Notably, the monotonic decrease in the electron-hole wave-function overlap with emission energy is found to be a very general and robust result for a large range of different parameters. The generality of this result can be understood in a simple physical picture and is related to the differences in the electron and heavy-hole effective masses. Thus, for any indium mole fraction, we have from Eqs. (A10)-(A12) that $m_{h h, b}>m_{e, b}$ and $m_{h h, x}, m_{h h, z}>m_{e}$. Increasing the emission energy corresponds to decreasing the QD size. The large QDs emitting at small energies have a relatively large electron-hole wavefunction overlap. Decreasing the QD size (i.e., increasing the emission energy) compresses both electron and hole wave functions, but eventually the electron wave functions is partially expelled from the QD so that the wave-function overlap is decreased. This effect is illustrated in Fig. 12, where the calculated electron and hole wave functions for two different QD sizes are plotted. It is clearly observed that a reduction in the QD height leads to a compression of the hole envelope wave function, while the electron wave function extends further into the surrounding GaAs barrier.

Finally, we want to stress a subtlety in the interpretation of the overlap integral. Contrary to what the name could suggest, the overlap integral is not equal to the probability of measuring the electron and hole at the same point in space. In our model, that probability shows in fact the opposite 
frequency dependence compared to the overlap integral. This agrees with theoretical results in the weak confinement regime. ${ }^{50}$ For very large QDs, the difference in effective masses has a negligible effect on the wave functions and the overlap integral is unity. When decreasing the QD size, the effective-mass difference becomes significant, thus, reducing the overlap integral and this determines the frequency dependence of the radiative decay rate of QDs in a homogeneous medium.

\section{CONCLUSION}

We have presented time-resolved measurements of spontaneous emission from self-assembled QDs near a semiconductor-air interface. The interface leads to a modification of the LDOS, which can be calculated in an exact model. The excellent agreement between theory and experiment enables a separation of the radiative and nonradiative decay rates, whereby they can be determined without any adjustable parameters and with unprecedented accuracy. We derived the theory behind the experiment by calculating the spontaneous emission radiative decay rate in a full quantum model in which spontaneous emission is described by Wigner-Weisskopf theory. The radiative decay rate is proportional to the projected LDOS, which was calculated using the Green's function formalism.

From our measurements of the radiative decay rate at different emission energies, we extracted the frequency dependence of the overlap of the electron and hole envelope wave functions. The experimental data were compared to theory by solving the effective-mass equation numerically for a QD potential, including the effects of shear and hydrostatic strain. From this model, the spontaneous emission spectrum, which is inhomogeneously broadened due to the different sizes of QDs making up the ensemble, and the electron-hole wave-function overlap were calculated. An attempt to model the emission spectrum using the QD height distribution obtained by AFM on uncapped QDs was unsuccessful leading to the conclusion that this height distribution does not properly reflect the nanoscopic confinement potential of overgrown QDs. Regarding the frequency dependence of the electron-hole wave-function overlap, we found good agreement between experiment and theory with reasonable assumptions about QD size, geometry, strain, and wetting-layer thickness assuming purely hydrostatic strain or no strain at all. In contrast, systematic deviations were found when including both shear and hydrostatic strain.

The frequency dependence of the wave-function overlap can be understood in terms of a simple physical picture. The larger hole mass leads to a larger compressibility of the hole wave function as compared to the electron so for smaller quantum dots (higher emission frequencies), the hole can be further compressed while the electron is partly expelled from the QD, thus, reducing the overlap. Although the numerical model employed here is too simple to reflect the nanoscopic details of the QD geometry such as, e.g., indium-gallium intermixing, it reflects this simple physical picture very well. A more detailed comparison between experiment and theory is limited by the lack of experimental input on the QD struc- ture at the atomic scale, which would be required to verify more sophisticated QD models. Also, we have shown by a simple scaling analysis that the nonradiative decay is likely due to nonradiative recombination at lattice defects near the QD-GaAs interface.

Combining the detailed optical experiments presented here with techniques to extract local material properties of QDs, e.g., by high-resolution transmission electron microscopy, will be a very exciting future research direction that also will pinpoint the need for more involved theoretical models of the QDs. We believe that the technique presented here to directly access the light-matter coupling strength will have important applications regarding proper design and characterization of solid-state quantum photonic devices.

\section{ACKNOWLEDGMENTS}

We wish to thank J. Højberg, A. Kreiner-Møller, and J. E. Mortensen for valuable work on the numerical model and $\mathrm{C}$. B. Sørensen for growth of the semiconductor material. We gratefully acknowledge the Danish Research Agency for financial support (Projects No. FNU 272-05-0083, No. FNU 272-06-0138, and No. FTP 274-07-0459).

\section{APPENDIX A: INFLUENCE OF STRAIN ON EXCITONS CONFINED IN QUANTUM DOTS}

Strain due to the lattice-constant mismatch between InAs and $\mathrm{GaAs}$ is responsible for the formation of QDs during MBE growth in the Stranski-Krastranov growth mode. This means that the QDs are highly strained and this has significant impact on the electronic band structure. The interplay between geometry, chemical composition, and strain is complicated for QDs. During the growth, diffusion of In and Ga takes place, so that the resulting QD will consist of a significant fraction of Ga even if it is grown by pure InAs. ${ }^{47}$ Furthermore, it has been reported that In is mainly concentrated in an inverted cone inside the QD giving rise to a complex strain profile. ${ }^{48}$ Complete knowledge about such complex details is still lacking, and the purpose here is to introduce simple strain models and judge their validity by comparing to experimental data. We consider a lens-shaped QD with a lateral extension, which is larger than the extension in the growth direction. For this reason, we approximate the strain of a QD by the model used in the case of a quantum well. This is further motivated by the fact that the QD is placed on top of a wetting layer (cf. Fig. 8). The strain modifies the band offsets and energy gap of the QD, and the valence-band degeneracy is lifted so that the transition with the lowest energy involves only heavy holes. This is illustrated in Fig. 13. We will assume that the bulk GaAs surrounding the QD is unstrained, however, only heavy-hole bands are included here as well since the influence of band mixing is minor in the barrier where the electron and hole wave functions are strongly damped.

One effect of strain is to shift the conduction and valence bands in energy. The strain describes the compression or expansion of the crystal lattice, which in general is described by a tensor $\epsilon_{n m}$. For biaxial strain, which describes the strain 
(a)

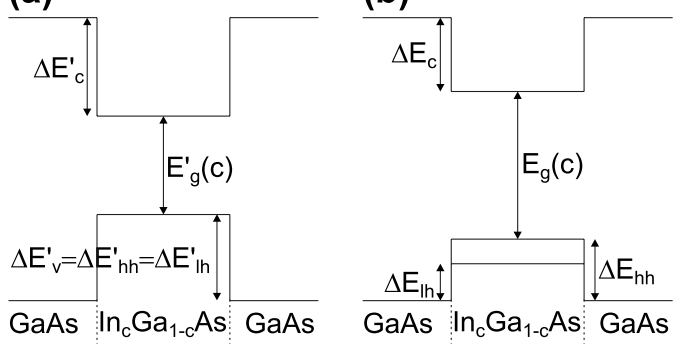

FIG. 13. Schematic illustration of the effect of strain on the band energies at $\mathbf{q}=\mathbf{0}$. (a) No strain and (b) biaxial strain. Biaxial strain shifts both the conduction band and the heavy-hole valence band closer to the continuum. The net effect is an increase in the band gap and therefore in the transition energy. Importantly, the degeneracy of the light and heavy-hole states is lifted and the lowestenergy transition involves heavy holes only.

at planar heterojunctions, only the diagonal elements are relevant. We consider a thin layer of $\operatorname{In}_{c} \mathrm{Ga}_{1-c}$ As with lattice constant $a_{\mathrm{QD}}$ embedded in a GaAs barrier with lattice constant $a_{b}$. We assume that all strain is incorporated in the $\mathrm{In}_{c} \mathrm{Ga}_{1-c} \mathrm{As}$ layer and since $a_{\mathrm{QD}}>a_{b}$ the strain will be compressive. We have ${ }^{19}$

$$
\epsilon_{x x}=\epsilon_{y y}=\frac{a_{b}-a_{\mathrm{QD}}}{a_{\mathrm{QD}}} \quad \epsilon_{z z}=-\frac{2 C_{12}}{C_{11}} \epsilon_{x x},
$$

where $C_{11}$ and $C_{12}$ are the elastic stiffness constants (matrix elements of the stiffness tensor). These strain components lead to a change in band structure and thus a modification of band energies and effective masses, as described by the Pikus-Bir strain model. ${ }^{19}$ Biaxial strain can be decomposed into components of hydrostatic and shear strain. The hydrostatic compressive strain of the QD leads to a decrease in the band offsets exactly as for any hydrostatic compressive strain resulting from, e.g., a decrease in the temperature. For a thin-strained epitaxial layer, the energy can be lowered by compensating the in-plane compressive strain by an expansion in the $z$ direction (shear strain). We obtain the following heavy-hole valence-/conduction-band offsets: ${ }^{19}$

$$
\begin{gathered}
\Delta E_{h h}=\Delta E_{v}^{\prime}-P_{\epsilon}-Q_{\epsilon}, \\
\Delta E_{c}=\Delta E_{c}^{\prime}-R_{\epsilon},
\end{gathered}
$$

where

$$
\begin{gathered}
P_{\epsilon}=a_{v}\left(\epsilon_{x x}+\epsilon_{y y}+\epsilon_{z z}\right), \\
Q_{\epsilon}=-\frac{b_{v}}{2}\left(\epsilon_{x x}+\epsilon_{y y}-2 \epsilon_{z z}\right), \\
R_{\epsilon}=a_{c}\left(\epsilon_{x x}+\epsilon_{y y}+\epsilon_{z z}\right),
\end{gathered}
$$

and we have introduced a number of quantities defined in Table I. $\Delta E_{c}^{\prime}\left(\Delta E_{v}^{\prime}\right)$ is the unstrained conduction(valence)band offset, which constitute $60 \%(40 \%)$ of the band-gap difference between bulk and $\mathrm{QD}$, so that, e.g., $\Delta E_{c}^{\prime}$ $=0.6\left[E_{g}^{\prime}(0)-E_{g}^{\prime}(c)\right]$. The band gap of the strained QD is given by

$$
E_{g}(c)=E_{g}^{\prime}(c)+P_{\epsilon}+Q_{\epsilon}+R_{\epsilon},
$$

where $E_{g}^{\prime}(c)$ is the unstrained band gap of the material. These effects on the band structure are illustrated in Fig. 13.

Another important consequence of strain is that the effective heavy-hole mass becomes highly anisotropic. In contrast, the effective electron mass is not modified considerably since the conduction band is much more energetically isolated than the valence bands. ${ }^{19,52}$ In our experiments, the growth direction $(z)$ is parallel to the [001] crystal axis, which is perpendicular to the wetting-layer plane $(x, y)$ ori-

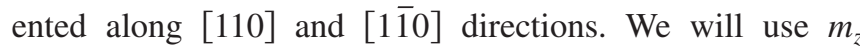
$=m_{\perp}=m_{[001]}$ and $m_{x}=m_{\|}=m_{[110]}$. Furthermore, since the crystal structure along the $[110]$ and $[1 \overline{1} 0]$ axes is identical apart from a series of rotations, we have that $m_{x}=m_{y}$.

TABLE I. Material parameters for $\operatorname{In}_{c} \mathrm{Ga}_{1-c}$ As at cryogenic temperatures used in this work. CB and VB indicate conduction- and valence-band parameters, respectively.

\begin{tabular}{lcccc}
\hline \hline Quantity & & Value for $\mathrm{In}_{c} \mathrm{Ga}_{1-c} \mathrm{As}$ & Unit & Reference $(\mathrm{s})$ \\
\hline Lattice constant & $a$ & $5.6503+0.4050 c$ & $\AA$ & 51 \\
Band gap & $E_{g}$ & $1.515-1.580 c+0.475 c^{2}$ & $\mathrm{eV}$ & 51 \\
CB effective mass & $m_{e}$ & $0.0667-0.0419 c-0.00254 c^{2}$ & $m_{0}$ & 51 \\
Luttinger parameter & $\gamma_{1}$ & $1 /[(1-c) / 6.98+c / 20.0]$ & & 51 and 52 \\
Luttinger parameter & $\gamma_{2}$ & $1 /[(1-c) / 2.06+c / 8.5]$ & & 51 and 52 \\
Luttinger parameter & $\gamma_{3}$ & $1 /[(1-c) / 2.93+c / 9.2]$ & & 51 and 52 \\
CB hydrostatic def. pot. & $a_{c}$ & $-8.013+2.933 c$ & $\mathrm{eV}$ & 51 \\
VB hydrostatic def. pot. & $a_{v}$ & $-1.824+0.024 c$ & $\mathrm{eV}$ & 51 \\
VB shear def. pot. & $b_{v}$ & $-2.0+0.2 c$ & $\mathrm{eV}$ & 52 \\
Elastic stiffness constant & $C_{11}$ & $1221-388.1 c$ & $\mathrm{GPa}$ & 52 \\
Elastic stiffness constant & $C_{12}$ & $566-113.4 c$ & $\mathrm{GPa}$ & 52 \\
Static dielectric constant & $\epsilon_{r}$ & $13.18+1.42 c$ & & 51 \\
Kane energy & $E_{p}(c)$ & $28.8-7.3 c$ & $\mathrm{eV}$ & 29 and 52 \\
\hline \hline
\end{tabular}


The heavy-hole masses for unstrained bulk $\operatorname{In}_{c} \mathrm{Ga}_{1-c} \mathrm{As}$ are given by

$$
\begin{gathered}
m_{h h, \|, b}^{\prime}=\frac{2}{2 \gamma_{1}-\gamma_{2}-3 \gamma_{3}}, \\
m_{h h, \perp, b}^{\prime}=\frac{1}{\gamma_{1}-2 \gamma_{2}},
\end{gathered}
$$

where $\gamma_{1}, \gamma_{2}$, and $\gamma_{3}$ are Luttinger parameters. ${ }^{52}$ These are listed along with all other relevant QD material parameters for this work in Table I. For $\operatorname{In}_{c} \mathrm{Ga}_{1-c}$ As, we have $\gamma_{2} \approx \gamma_{3}$ allowing for the axial approximation. Here $\gamma_{2}$ and $\gamma_{3}$ are replaced by their average value $\bar{\gamma}=\left(\gamma_{2}+\gamma_{3}\right) / 2$, leading to an isotropic heavy-hole mass valid for unstrained bulk semiconductors $^{53}$

$$
m_{h h, \|, b}^{\prime}=m_{h h, \perp, b}=\frac{1}{\gamma_{1}-2 \bar{\gamma}} .
$$

These bulk effective masses are used to describe the GaAs barrier material surrounding the QDs. The strained heavyhole effective masses in the directions parallel with and perpendicular to the wetting-layer plane are given by ${ }^{19,29,53}$

$$
\begin{aligned}
m_{h h, \|, \mathrm{QD}} & =\frac{1}{\gamma_{1}+\bar{\gamma}}, \\
m_{h h, \perp, \mathrm{QD}} & =\frac{1}{\gamma_{1}-2 \bar{\gamma}},
\end{aligned}
$$

showing that the parallel component is strongly modified by strain.

\section{APPENDIX B: NUMERICAL MODELING OF ENVELOPE WAVE FUNCTIONS}

We solve the effective-mass equation for electrons in the conduction band and holes in the valence band in order to calculate the overlap of the envelope wave functions. The effective-mass equations describing the electron and the hole have the same form, but the effective hole mass anisotropy must be taken into account. We therefore consider the anisotropic valence-band problem, which has the isotropic conduction-band problem as a special case. For both electrons and holes, the effective mass depends on position due to the different effective masses in the QD and in the surrounding crystal matrix.
Using cylindrical coordinates $(\rho, \phi, z)$ and the axial approximation, the kinetic term in Eq. (2) reads as

$$
\begin{aligned}
H_{\text {kin }}= & -\frac{\hbar^{2}}{2 m_{0}}\left[\frac{1}{\rho} \frac{\partial}{\partial \rho}\left(\frac{\rho}{m_{n \|}(\rho, z)} \frac{\partial}{\partial \rho}\right)+\frac{1}{m_{n \|}(\rho, z) \rho^{2}} \frac{\partial^{2}}{\partial \phi^{2}}\right. \\
& \left.+\frac{\partial}{\partial z}\left(\frac{1}{m_{n \perp}(\rho, z)} \frac{\partial}{\partial z}\right)\right] .
\end{aligned}
$$

Using separation of variables $F(\mathbf{r})=R(\rho, z) \Phi(\phi)$, the effective-mass Schrödinger equation can be written as

$$
\begin{aligned}
\frac{-1}{\Phi(\phi)} \frac{\partial^{2}}{\partial \phi^{2}} \Phi(\phi)= & \frac{\rho}{R(\rho, z)} \frac{\partial}{\partial \rho} R(\rho, z) \\
& +\frac{m_{n \|}(\rho, z) \rho^{2}}{R(\rho, z)} \frac{\partial}{\partial \rho}\left[\frac{1}{m_{n \|}(\rho, z)} \frac{\partial}{\partial \rho} R(\rho, z)\right] \\
& +\frac{m_{n \|}(\rho, z) \rho^{2}}{R(\rho, z)} \frac{\partial}{\partial z}\left[\frac{1}{m_{n \perp}(\rho, z)} \frac{\partial}{\partial z} R(\rho, z)\right] \\
& +\frac{2 m_{0} m_{n \|}(\rho, z) \rho^{2}}{\hbar^{2}}[V(\rho, z)-E] .
\end{aligned}
$$

The left- and right-hand sides of this equation are independent and they must therefore equal a constant, i.e., $\frac{1}{\Phi(\phi)} \frac{\partial^{2}}{\partial \phi^{2}} \Phi(\phi)=-l^{2}$. The solution of this equation is $\Phi(\phi)$ $=c_{1} \cos (l \phi)+c_{2} \sin (l \phi)$, which by the boundary condition $\Phi(0)=\Phi(2 \pi)$ implies that $l$ must be an integer. We are considering the ground-state transition and therefore take $l=0$. This leaves an equation describing the electronic motion in the $(\rho, z)$ plane.

Equation (B2) is solved numerically after being reduced to a dimensionless form in order to avoid numerical issues related to the very small factors $\left(\propto \hbar^{2}\right)$ appearing in this equation. We define the new dimensionless quantities through $\rho$ $=k_{\rho} \tilde{\rho}, z=k_{z} \widetilde{z}, R(\rho, z)=k_{R} \widetilde{R}(\widetilde{\rho}, \widetilde{z}), V(\rho, z)=V_{0} \widetilde{V}(\widetilde{\rho}, \widetilde{z}), E=V_{0} \widetilde{E}$, and $V_{0}=\hbar^{2} /\left(2 m_{0} k_{\rho}^{2}\right)$, and furthermore take $k_{\rho}=k_{z}=1 \mathrm{~nm}$ so that all spatial dimensions are measured in units of nanometer. By this transformation, we obtain

$$
\begin{aligned}
& \frac{1}{m_{\|}(\tilde{\rho}, \widetilde{z}) \tilde{\rho}} \frac{\partial}{\partial \widetilde{\rho}} \widetilde{R}(\widetilde{\rho}, \widetilde{z})+\frac{\partial}{\partial \widetilde{\rho}}\left[\frac{1}{m_{\|}(\widetilde{\rho}, \widetilde{z})} \frac{\partial}{\partial \widetilde{\rho}} \widetilde{R}(\widetilde{\rho}, \widetilde{z})\right] \\
& +\frac{\partial}{\partial \widetilde{z}}\left[\frac{1}{m_{\perp}(\widetilde{\rho}, \widetilde{z})} \frac{\partial}{\partial \widetilde{z}} \tilde{R}(\tilde{\rho}, \widetilde{z})\right]+[\tilde{V}(\tilde{\rho}, \tilde{z}) \\
& \left.-\frac{l^{2}}{m_{\|}(\widetilde{\rho}, \widetilde{z}) \widetilde{\rho}^{2}}\right] \tilde{R}(\tilde{\rho}, \widetilde{z})=\tilde{E} \widetilde{R}(\tilde{\rho}, \tilde{z}),
\end{aligned}
$$

which is solved numerically using a finite element method.

\footnotetext{
*ssto@fotonik.dtu.dk

†pelo@fotonik.dtu.dk; http://www.fotonik.dtu.dk/quantumphotonics

${ }^{1}$ A. Imamoglu, D. D. Awschalom, G. Burkard, D. P. DiVincenzo, D. Loss, M. Sherwin, and A. Small, Phys. Rev. Lett. 83, 4204 (1999).

${ }^{2}$ J. M. Gérard, B. Sermage, B. Gayral, B. Legrand, E. Costard, and V. Thierry-Mieg, Phys. Rev. Lett. 81, 1110 (1998).
}

${ }^{3}$ J. P. Reithmaier, G. Sęk, A. Löffler, C. Hofmann, S. Kuhn, S. Reitzenstein, L. V. Keldysh, V. D. Kulakovskii, T. L. Reinecke, and A. Forchel, Nature (London) 432, 197 (2004).

${ }^{4}$ T. Yoshie, A. Scherer, J. Hendrickson, G. Khitrova, H. M. Gibbs, G. Rupper, C. Ell, O. B. Shchekin, and D. G. Deppe, Nature (London) 432, 200 (2004).

${ }^{5}$ C. Kistner, T. Heindel, C. Schneider, A. Rahimi-Iman, S. Re- 
itzenstein, S. Höfling, and A. Forchel, Opt. Express 16, 15006 (2008).

${ }^{6}$ A. Laucht, F. Hofbauer, N. Hauke, J. Angele, S. Stobbe, M. Kaniber, G. Böhm, P. Lodahl, M.-C. Amann, and J. J. Finley, New J. Phys. 11, 023034 (2009).

${ }^{7}$ P. Michler, A. Kiraz, C. Becher, W. V. Schoenfeld, P. M. Petroff, L. Zhang, E. Hu, and A. Imamoglu, Science 290, 2282 (2000).

${ }^{8}$ T. Lund-Hansen, S. Stobbe, B. Julsgaard, H. Thyrrestrup, T. Sünner, M. Kamp, A. Forchel, and P. Lodahl, Phys. Rev. Lett. 101, 113903 (2008).

${ }^{9}$ S. Reitzenstein, T. Heindel, C. Kistner, A. Rahimi-Iman, C. Schneider, S. Höfling, and A. Forchel, Appl. Phys. Lett. 93, 061104 (2008).

${ }^{10}$ P. Lodahl, A. Floris van Driel, I. S. Nikolaev, A. Irman, K. Overgaag, D. Vanmaekelbergh, and W. L. Vos, Nature (London) 430, 654 (2004).

${ }^{11}$ B. Julsgaard, J. Johansen, S. Stobbe, T. Stolberg-Rohr, T. Sünner, M. Kamp, A. Forchel, and P. Lodahl, Appl. Phys. Lett. 93, 094102 (2008).

${ }^{12}$ I. Robert, E. Moreau, B. Gayral, J.-M. Gerard, and I. Abram, Physica E 13, 606 (2002).

${ }^{13}$ X. Brokmann, L. Coolen, M. Dahan, and J. P. Hermier, Phys. Rev. Lett. 93, 107403 (2004).

${ }^{14}$ M. D. Leistikow, J. Johansen, A. J. Kettelarij, P. Lodahl, and W. L. Vos, Phys. Rev. B 79, 045301 (2009).

${ }^{15}$ J. Johansen, S. Stobbe, I. S. Nikolaev, T. Lund-Hansen, P. T. Kristensen, J. M. Hvam, W. L. Vos, and P. Lodahl, Phys. Rev. B 77, 073303 (2008).

${ }^{16}$ E. M. Purcell, Phys. Rev. 69, 681 (1946).

${ }^{17}$ K. H. Drexhage, J. Lumin. 1-2, 693 (1970).

${ }^{18}$ L. Novotny and B. Hecht, Principles of Nano-Optics (Cambridge University Press, New York, 2007).

${ }^{19}$ S. L. Chuang, Physics of Optoelectronic Devices (Wiley, New York, 1995).

${ }^{20}$ R. R. Chance, A. Prock, and R. Silbey, Adv. Chem. Phys. 37, 1 (1978).

${ }^{21}$ D. Birkedal, J. Bloch, J. Shah, L. N. Pfeiffer, and K. West, Appl. Phys. Lett. 77, 2201 (2000)

${ }^{22}$ R. J. Warburton, C. S. Dürr, K. Karrai, J. P. Kotthaus, G. Medeiros-Ribeiro, and P. M. Petroff, Phys. Rev. Lett. 79, 5282 (1997).

${ }^{23}$ K. L. Silverman, R. P. Mirin, S. T. Cundiff, and A. G. Norman, Appl. Phys. Lett. 82, 4552 (2003).

${ }^{24}$ C. F. Wang, A. Badolato, I. Wilson-Rae, P. M. Petroff, E. Hu, J. Urayama, and A. Imamoglu, Appl. Phys. Lett. 85, 3423 (2004).

${ }^{25}$ N. C. Barford, Experimental Measurements: Precision, Error and Truth (Wiley, New York, 1990).

${ }^{26}$ J. Johansen, B. Julsgaard, S. Stobbe, J. M. Hvam, and P. Lodahl arXiv:0905.4493 (unpublished).

${ }^{27}$ S. Cortez, O. Krebs, P. Voisin, and J.-M. Gérard, Phys. Rev. B 63, 233306 (2001).
${ }^{28}$ A. F. van Driel, G. Allan, C. Delerue, P. Lodahl, W. L. Vos, and D. Vanmaekelbergh, Phys. Rev. Lett. 95, 236804 (2005).

${ }^{29}$ L. A. Coldren and S. W. Corzine, Diode Lasers and Photonic Integrated Circuits (Wiley, New York, 1995).

${ }^{30}$ E. C. Le Ru, J. Fack, and R. Murray, Phys. Rev. B 67, 245318 (2003).

${ }^{31}$ P. A. Dalgarno, J. M. Smith, J. McFarlane, B. D. Gerardot, K. Karrai, A. Badolato, P. M. Petroff, and R. J. Warburton, Phys. Rev. B 77, 245311 (2008).

${ }^{32}$ E. Hanamura, Phys. Rev. B 37, 1273 (1988).

${ }^{33}$ R. V. N. Melnik and M. Willatzen, Nanotechnology 15, 1 (2004).

${ }^{34}$ T. Markussen, P. Kristensen, B. Tromborg, T. W. Berg, and J. Mørk, Phys. Rev. B 74, 195342 (2006).

${ }^{35}$ M. G. Burt, J. Phys.: Condens. Matter 4, 6651 (1992).

${ }^{36}$ C. Pryor, Phys. Rev. B 57, 7190 (1998).

${ }^{37}$ P. Kristensen, A. F. Koenderink, P. Lodahl, B. Tromborg, and J. Mørk, Opt. Lett. 33, 1557 (2008).

${ }^{38}$ S. S. Li, J. B. Xia, Z. L. Yuan, Z. Y. Xu, W. Ge, X. R. Wang, Y. Wang, J. Wang, and L. L. Chang, Phys. Rev. B 54, 11575 (1996).

${ }^{39}$ H. Haug and S. W. Koch, Quantum Theory of the Optical and Electronic Properties of Semiconductors (World Scientific, Singapore, 1994).

${ }^{40}$ N. Vats, S. John, and K. Busch, Phys. Rev. A 65, 043808 (2002).

${ }^{41} \mathrm{R}$. Loudon, The Quantum Theory of Light (Oxford University Press, New York, 2000).

${ }^{42}$ H. T. Dung, L. Knöll, and D.-G. Welsch, Phys. Rev. A 62, 053804 (2000).

${ }^{43}$ M. Paulus, P. Gay-Balmaz, and O. J. F. Martin, Phys. Rev. E 62, 5797 (2000).

${ }^{44}$ J. H. Davies, The Physics of Low-Dimensional Semiconductors: An Introduction (Cambridge University Press, New York, 1998).

${ }^{45}$ A. D. Andreev and E. P. O'Reilly, Appl. Phys. Lett. 87, 213106 (2005).

${ }^{46}$ Note that in Fig. 3.B of Ref. 15, the theoretical curve plotted is mistakenly $\left|\left\langle F_{h}(\omega) \mid F_{e}(\omega)\right\rangle\right|$ rather than $\left|\left\langle F_{h}(\omega) \mid F_{e}(\omega)\right\rangle\right|^{2}$.

${ }^{47}$ I. Kegel, T. H. Metzger, A. Lorke, J. Peisl, J. Stangl, G. Bauer, J. M. García, and P. M. Petroff, Phys. Rev. Lett. 85, 1694 (2000).

${ }^{48}$ N. Liu, J. Tersoff, O. Baklenov, A. L. Holmes, Jr., and C. K. Shih, Phys. Rev. Lett. 84, 334 (2000).

${ }^{49}$ M. De Giorgi, A. Passaseo, R. Rinaldi, T. Johal, R. Cingolani, A. Taurino, M. Catalano, and P. Crozier, Phys. Status Solidi B 224 17 (2001).

${ }^{50}$ T. Takagahara, Phys. Rev. B 47, 4569 (1993).

${ }^{51}$ O. Stier, M. Grundmann, and D. Bimberg, Phys. Rev. B 59, 5688 (1999).

${ }^{52}$ I. Vurgaftman, J. R. Meyer, and L. R. Ram-Mohan, J. Appl. Phys. 89, 5815 (2001).

${ }^{53}$ W. W. Chow and S. W. Koch, Semiconductor-Laser Fundamentals (Springer, New York, 1999). 